\title{
In situ epitaxial engineering of graphene and h-BN lateral heterostructure with a tunable morphology comprising h-BN domains
}

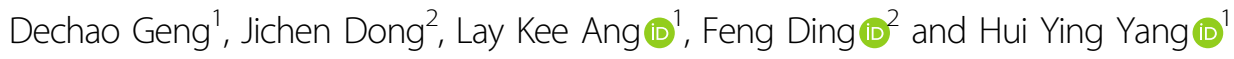

\begin{abstract}
Graphene and hexagonal boron nitride (h-BN), as typical two-dimensional (2D) materials, have long attracted substantial attention due to their unique properties and promise in a wide range of applications. Although they have a rather large difference in their intrinsic bandgaps, they share a very similar atomic lattice; thus, there is great potential in constructing heterostructures by lateral stitching. Herein, we present the in situ growth of graphene and h-BN lateral heterostructures with tunable morphologies that range from a regular hexagon to highly symmetrical star-like structure on the surface of liquid Cu. The chemical vapor deposition (CVD) method is used, where the growth of the h$\mathrm{BN}$ is demonstrated to be highly templated by the graphene. Furthermore, large-area production of lateral G-h-BN heterostructures at the centimeter scale with uniform orientation is realized by precisely tuning the CVD conditions. We found that the growth of h-BN is determined by the initial graphene and symmetrical features are produced that demonstrate heteroepitaxy. Simulations based on the phase field and density functional theories are carried out to elucidate the growth processes of G-h-BN flakes with various morphologies, and they have a striking consistency with experimental observations. The growth of a lateral G-h-BN heterostructure and an understanding of the growth mechanism can accelerate the construction of various heterostructures based on 2D materials.
\end{abstract}

\section{Introduction}

Conventional heteroepitaxy refers to the formation of an extended single-crystal film on the two-dimensional (2D) surface of a single crystal of a different material, as exemplified by the growth of $\mathrm{GaP}$ on $\mathrm{Si}^{1,2}$. In general, the atomic lattice of the surface can act as a template for the initial growth of the epilayer if the different materials have similar lattice constants. As two typical 2D materials, graphene $(\mathrm{G})$ and hexagonal boron nitride (h-BN), have fueled enormous interest; importantly, they have similar crystal structures with a lattice constant mismatch of only $2 \%$ and possess great promise in constructing in-plane heterostructures via heteroepitaxial growth ${ }^{3,4}$. In-plane

\footnotetext{
Correspondence: Feng Ding (f.ding@unist.ac.kr) or Hui

Ying Yang (yanghuiying@sutd.edu.sg)

${ }^{1}$ Pillar of Engineering Product Development, Singapore University of Technology and Design, 8 Somapah Road, 487372 Singapore, Singapore

${ }^{2}$ Centre for Multidimensional Carbon Materials, Institute for Basic Science, Ulsan, Korea

These authors contributed equally: Dechao Geng, Jichen Dong
}

heterostructures have been generated by lithographic approaches ${ }^{5}$ and the regrowth of graphene around patterned $\mathrm{h}-\mathrm{BN}^{6}$. In these approaches, the patterning step has the potential to induce unwanted structural and chemical disorder. To avoid this complication, direct growth of in-plane heterostructures has also been investigated. In the past few years, the construction of lateral heterostructures of $\mathrm{G}$ and h-BN on metals by chemical vapor deposition $(\mathrm{CVD})$ has been realized ${ }^{7-11}$. However, the scale of those heterostructures is still limited to micrometers, hindering further applications. Moreover, the detailed growth mechanism remains elusive, and detailed insights are highly desired. Given the interactions in three-dimensional space between the 2D graphene seed crystal and the h-BN surroundings, determining how the h-BN crystal grows from the one-dimensional (1D) edge of the graphene seed crystal (or vice versa) to form a lateral heterostructure is not a trivia task.

\section{(c) The Author(s) 2019}

(c) (i) Open Access This article is licensed under a Creative Commons Attribution 4.0 International License, which permits use, sharing, adaptation, distribution and reproduction cc) in any medium or format, as long as you give appropriate credit to the original author(s) and the source, provide a link to the Creative Commons license, and indicate if changes were made. The images or other third party material in this article are included in the article's Creative Commons license, unless indicated otherwise in a credit line to the material. If material is not included in the article's Creative Commons license and your intended use is not permitted by statutory regulation or exceeds the permitted use, you will need to obtain permission directly from the copyright holder. To view a copy of this license, visit http://creativecommons.org/licenses/by/4.0/. 
Here, we report the in situ growth of graphene and h$\mathrm{BN}$ lateral heterostructures with a tunable morphology on a liquid $\mathrm{Cu}$ surface by the CVD method, where the highly templated growth of $\mathrm{h}-\mathrm{BN}$ induced by pregrown graphene crystals is demonstrated. During the whole two-step growth process, hexagonal graphene crystals are first fabricated, and then, h-BN growth is conducted. Largearea G-h-BN heterostructures are produced on the whole substrate with a uniform orientation. It is found that the subsequent growth of the h-BN is templated by the inner graphene, resulting in morphology engineering of the hBN by modulating the CVD conditions. Furthermore, the as-grown $\mathrm{h}$ - $\mathrm{BN}$ with a variety of morphologies is highly symmetrical in structure and demonstrates heteroepitaxy. Theoretical calculations based on phase field theory (PFT) are conducted to probe the morphology evolution of the h-BN domains grown from a graphene seed crystal and the results are consistent with the experimental observations.

\section{Results and discussion}

The growth procedure is schematically illustrated in Fig. 1a and described in detail in the Experimental section. First, monolayer graphene crystals were grown by ambient pressure CVD on a liquid $\mathrm{Cu}$ surface. Notably, liquid $\mathrm{Cu}$ has been recognized as an effective catalyst in tuning the size and morphology of graphene $\operatorname{crystals}^{12,13}$, whereas in this case, large-area regular hexagonal graphene crystals with similar sizes were first prepared (Fig. S1) and provided a template in $2 \mathrm{D}$ space. It should be noted that the as-grown hexagonal graphene domains were single crystals (Fig. S2). This step is analogous to the common practice of obtaining fresh starting surfaces for conventional epitaxy ${ }^{14,15}$ and is critical to the formation of the h-BN epilayer here. In the second stage, immediately after graphene growth, $\mathrm{CH}_{4}$ was introduced and h-BN growth was initiated under the same conditions, e.g., the same temperature and flow rate, with ammonia borane $\left(\mathrm{NH}_{3}-\mathrm{BH}_{3}\right)$ powder as the precursor. For controlled h-BN epilayer formation, we ensured that (i) the growth was indeed confined to $2 \mathrm{D}$ space, namely, the liquid $\mathrm{Cu}$ surface-and (ii) the growth was sufficiently slow to allow for epitaxial growth. A low precursor supply rate satisfied both requirements and was achieved by exploiting APCVD process kinetics ${ }^{3}$, which is analogous to graphene APCVD using a highly diluted $\mathrm{CH}_{4}$ precursor that yields crystallites with zigzag edges ${ }^{16-18}$.

The as-grown G-h-BN heterostructures are shown in Fig. 1b, c. Also, h-BN ribbons, referred to as "epistrips", which have a distinct contrast in SEM images, are observed along the whole six edges of the graphene hexagons. The coverage scale can be up to a centimeter on the whole $\mathrm{Cu}$ substrate, whereas the as-grown heterostructures have a uniform orientation and size (Figs.
S3-S5). Notably, the width of the epistrips can be tuned by controlling the growth time (Fig. S6). As clearly shown in Fig. 1c, a certain amount of white particles are aggregated on the edges of the h-BN epistrips. Energy dispersive X-ray spectroscopy measurements of the particles verified the presence of silicon oxides (Fig. S7). Given the required high temperature $\left(1100^{\circ} \mathrm{C}\right)$ to obtaina liquid $\mathrm{Cu}$ state, it is deduced that the silicon oxides are derived from the deposition of quartz tubes on the surface ${ }^{19}$ and prefer to reside on the edge sites, which show a high activity. This unique feature of the deposition can serve as an indicator of the surface reactivity. Furthermore, it is worth noting that all the G-h-BN flakes share a similar size, both inside the hexagonal $\mathrm{G}$ and outside the $\mathrm{h}-\mathrm{BN}$, indicating a uniform growth on the liquid $\mathrm{Cu}$ for the two materials during the CVD process. An electrochemical bubbling method was employed to transfer the samples onto a $\mathrm{SiO}_{2} / \mathrm{Si}$ substrate, as shown in Fig. 1d. The whole flake is well maintained, suggesting an intact transfer. Thickness measurements further show monolayer features for both the $\mathrm{G}$ and h-BN (Figs. S8 and S9).

For a single G-h-BN flake, morphology engineering was realized by precisely tuning the CVD conditions during the growth. A series of shaped flakes were fabricated, with the inner graphene part always displaying a hexagonal shape and the outer $\mathrm{h}-\mathrm{BN}$ part having various shapes. In our case, the growth conditions for graphene were always the same, allowing for regular growth of the hexagonal graphene crystals. The differences were in the gas flow rate and $\mathrm{h}-\mathrm{BN}$ growth time. The detailed growth conditions for various shaped G-h-BN flakes are listed in Table $\mathrm{S} 1$. At a very low flow rate of $\mathrm{H}_{2}$ during $\mathrm{h}-\mathrm{BN}$ growth, the as-grown $\mathrm{h}-\mathrm{BN}$ displayed typical ribbon shapes along the edges of the hexagonal graphene that finally formed hexagonal G-h-BN flakes (Fig. 2a). In this case, the very low flow rate ensured strictly epitaxial growth of the h-BN along the graphene edges and produced anisotropy, similar to the growth of compact hexagonal graphene ${ }^{17}$. With an increased flow rate, more concave polyhedral structures (Fig. 2b) were produced. It was reasonably speculated that the appearance of the concave morphology was due to the existence of grain boundaries between neighboring $\mathrm{h}-\mathrm{BN}$ grains, similar to the case for graphene growth $^{20,21}$. When the flow rate was further increased to $60 \mathrm{sccm}$, highly symmetrical star-like G-h-BN flakes were observed (Fig. 2c). For the three kinds of G-h-BN flakes, Raman mapping measurements were conducted. Figure $2 \mathrm{~d}-\mathrm{f}$ shows the corresponding Raman mapping images, where the inner graphene and outer h-BN profiles can be clearly distinguished due to the color contrast. Note that there is partial overlap for the $E_{2 g}$ peak for h-BN and $\mathrm{D}$ peak for graphene. The intensity is much higher for the inner part of the graphene than that for outer part of the h-BN. Based on the Raman spectrum, the monolayer 


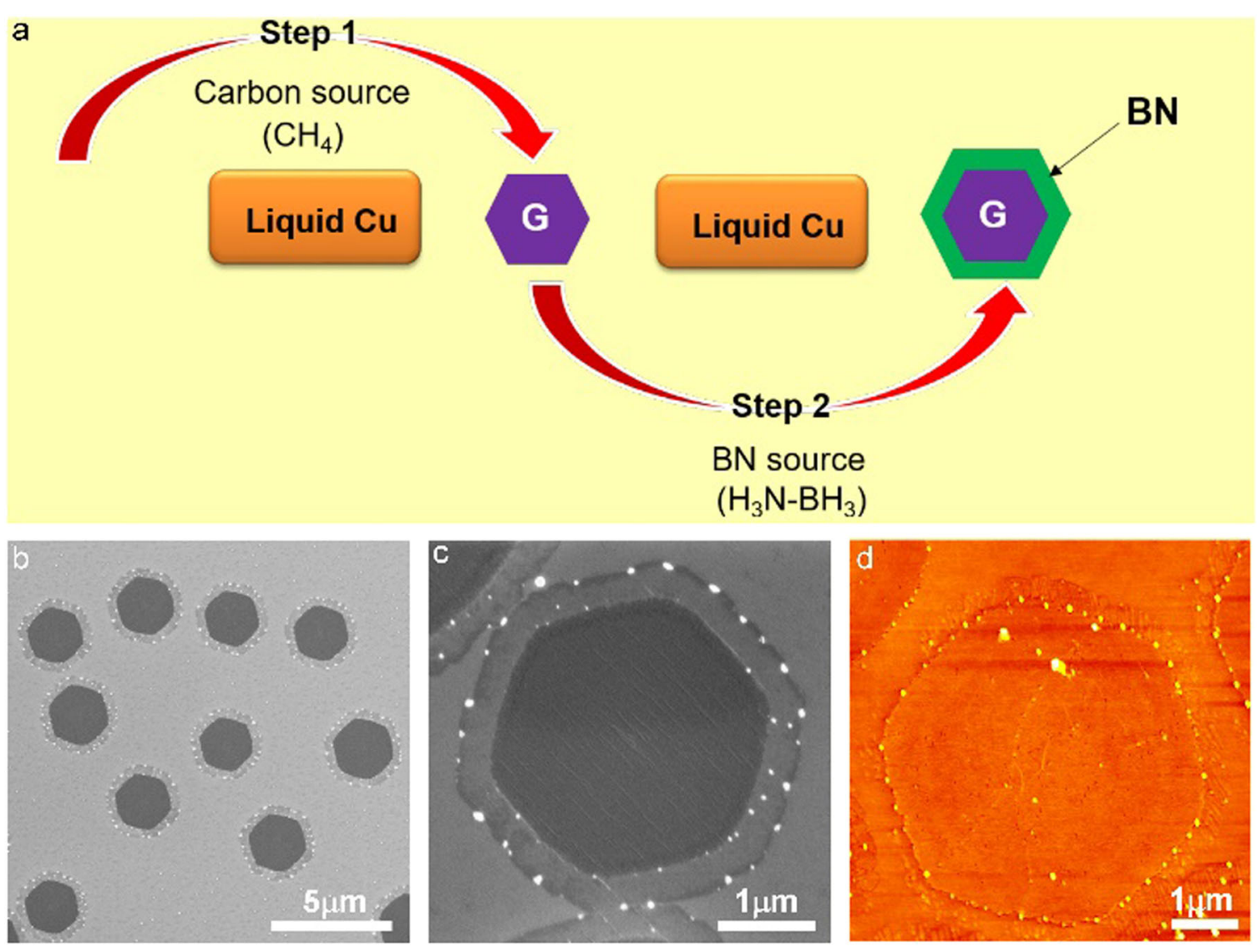

Fig. 1 Lateral heterostructure of graphene and $\mathbf{h}-\mathbf{B N}$. a Schematic of the two-step continuous growth of graphene and h-BN lateral heterostructure by CVD on a liquid Cu surface. b, c SEM images of large-area and single G-h-BN structures, respectively. Note that the subsequently grown h-BN has an epistrip morphology. d AFM image of a single G-h-BN flake

features for both graphene and h-BN were further confirmed, where the typical peaks were characterized for both monolayer graphene and h-BN (Fig. S10). Furthermore, the lateral heterostructure was verified by STM measurements, showing epitaxy between the inner graphene and h-BN (Figs. S11-S13).

Furthermore, the whole shape evolution of the three cases as a function of growth time were all directly observed, and the detailed growth conditions are listed in Table S2. For the first case, as shown in Fig. 3a-c, the outer h-BN ribbons exhibit a gradual expanded growth perpendicular to the six edges of the graphene with increasing time. Notably, the h-BN epistrips occur as branches along the edges, as seen in Fig. 3b, c. Based on the kinetic process of crystal growth, discrepancies in the growth rate along certain directions account for shape change $^{22,23}$. In our case, the shape evolution is attributed to unbalanced growth among neighboring $\mathrm{h}-\mathrm{BN}$ grains, as explained later. Figure $3 \mathrm{~d}-\mathrm{f}$ further illustrates the second growth evolution case for concave G-h-BN flakes, where the outer h-BN parts gradually evolved into six individual hexagonal flakes anchored on the corresponding six edges of graphene. It is deduced that the subsequent h-BN growth can be coincident with first graphene owing to the similar atomic lattice. With further increases in the gas flow rate, as marked in the third case, gradual evolution of flower-like G-h-BN flakes was also directly observed, as shown in Fig. 3g-i. Based on the above observations, for the three kinds of G-h-BN flakes, the outer h-BN growth was highly templated by the inner G first and gradually evolved into different morphologies under certain conditions. It should be noted that the images shown in Fig. 3 are not the same flakes in each line. During our CVD growth experiments, the samples were grown at different times and then characterized by SEM after cooling to room temperature. In situ SEM measurements are planned as a next step for a clear demonstration of the evolution process. The growth mechanism is elucidated in the following section.

Theoretical calculations were conducted to probe the whole growth process of the G-h-BN heterostructures. It was previously reported that hexagonal $\mathrm{G}$ crystals grown on metal substrates have zigzag $(\mathrm{ZZ})$ edges $^{18}$; since several experiments confirmed a $\mathrm{ZZ}$ edge at the interface of $\mathrm{G}$ 
a
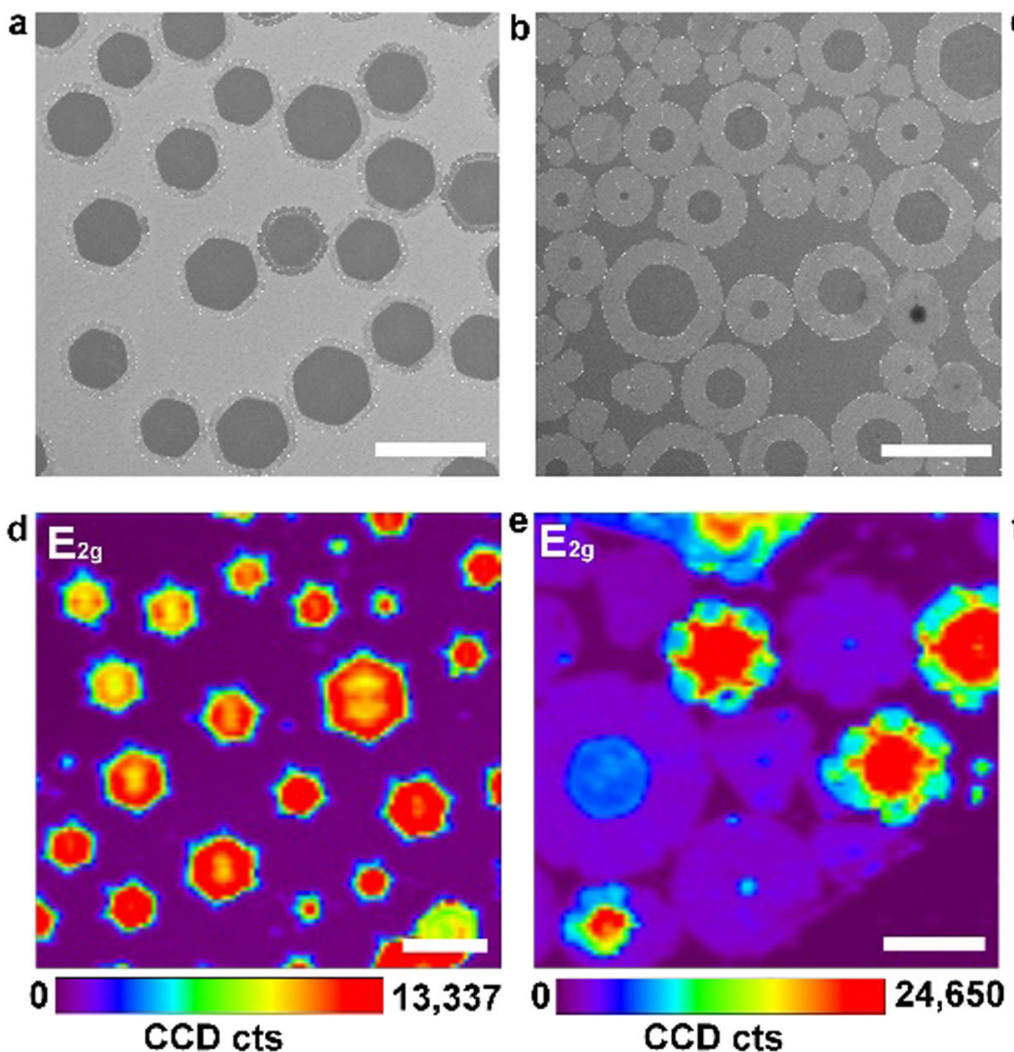
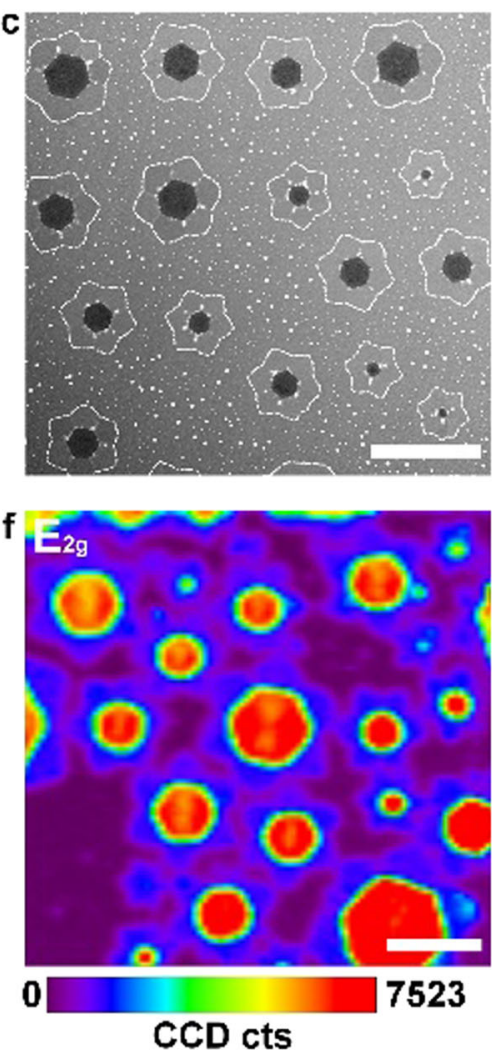

Fig. 2 Shape engineering of G-h-BN flakes on a liquid Cu surface. a Hexagonal G-h-BN flake. b Concave-shaped G-h-BN flake. c Flower-like G-hBN flake. $\mathbf{d}-\mathbf{f}$ Raman mapping images of the corresponding three shaped flakes for the $E_{2 g}$ peak at $1371 \mathrm{~cm}^{-1}$. All scale bars are $10 \mu \mathrm{m}$

and h-BN in-plane heterostructures ${ }^{9,24}$, it was assumed that in our case, the subsequent h-BN growth would be atomically grown along the $\mathrm{ZZ}$ edges of the G. Furthermore, it was shown that two bonded modes exist when h$\mathrm{BN}$ is atomically connected with graphene, where one is a $\mathrm{C}-\mathrm{N}$ bond and the other is a $\mathrm{C}-\mathrm{B}$ bond $^{25}$. In our case, the interface between the $\mathrm{G}$ flake and h-BN periphery was also investigated. It should be noted that hydrogen can serve as an important factor to affect the growth during CVD process. Hydrogen flow can affect the growth rates of the ZZN and ZZB edges in two ways. (i) The edges of the h-BN can be passivated by substrates under low $\mathrm{H}_{2}$ partial pressure and passivated by hydrogen under high $\mathrm{H}_{2}$ partial pressure ${ }^{26}$. Different passivations of the h-BN edges can result in different growth rates $^{27}$. (ii) The growth rates of the ZZB and ZZN edges are also affected by the chemical potentials and the concentrations of boron and nitrogen atoms on the $\mathrm{Cu}$ substrate. Boron and nitrogen atoms are obtained from the decomposition of borane $\left(\mathrm{NH}_{3}-\mathrm{BH}_{3}\right)$. However, due to the different solubility and therefore different binding energies of boron and nitrogen to the $\mathrm{Cu}$ substrates ${ }^{28}$, their concentrations change differently in the presence of hydrogen. This is because hydrogen can etch boron and nitrogen atoms on the $\mathrm{Cu}$ substrate. Therefore, the $\mathrm{H}_{2}$ partial pressure can effectively modulate the chemical potentials of boron and nitrogen (Fig. S14).

At the interface between the graphene and $\mathrm{h}-\mathrm{BN}$, the case where the $\mathrm{ZZ}$ graphene edge is bonded to the $\mathrm{ZZ}$ nitrogen (ZZN) edge of h-BN is first considered. Figure $4 a$ shows a schematic of the atomic structure near the interface between the $\mathrm{G}$ and h-BN. It can be clearly seen that the h-BN domain is actually composed of six grains due to the lower symmetry of h-BN than that of the graphene. The grain boundary (GB) between h-BN grains is formed by $\mathrm{B}-\mathrm{B}$ bonds along $\mathrm{ZZ}$ directions. To explore the growth behaviors of the G-h-BN flakes with this type of interface between the graphene and h-BN, simulations based on PFT were carried out (see SI for details). As shown in Fig. 4b1, a hexagonal domain (red color) with six strips (red color) is first built to represent the graphene grain and the newly grown $\mathrm{h}-\mathrm{BN}$ grains, respectively. Due to the threefold symmetry of the $\mathrm{h}-\mathrm{BN}$, its $\mathrm{ZZ}$ edges are classified into two categories, i.e., zigzag nitrogen (ZZN) and zigzag boron (ZZB). Therefore, the outer edges of $h$ BN grains shown in Fig. $4 \mathrm{~b} 1$ are ZZB edges.

Previous studies have already shown that the growth rates of ZZN and ZZB edges ( $R_{\mathrm{ZZN}}$ and $\mathrm{R}_{\mathrm{ZZB}}$, respectively) 

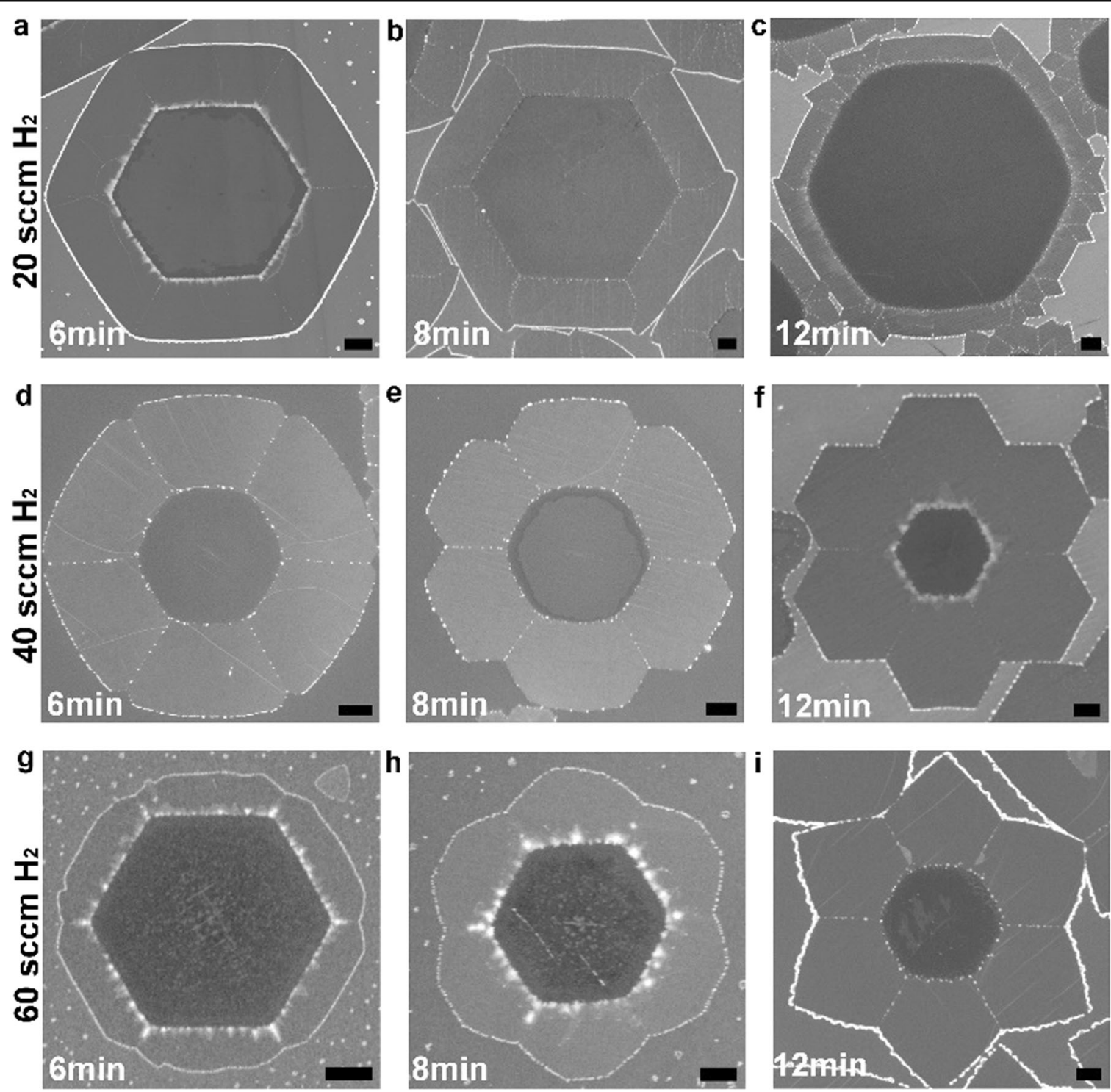

Fig. 3 The shape evolution of the three kinds G-h-BN flakes. a-c Shape evolution of hexagonal G-h-BN flake. $\mathbf{d}-\mathbf{f}$ Shape evolution of concave Gh-BN flakes. g-i Shape evolution of flower-like G-h-BN flakes. All scale bars are $1 \mu \mathrm{m}$

are different, and $R_{Z Z N}$ and $R_{Z Z B}$ can be effectively tuned. Consequently, h-BN grains with shapes ranging from a hexagon, truncated triangle to triangle can be formed $^{29-32}$. Here, we first assume that $R_{\mathrm{ZZN}}: R_{\mathrm{ZZB}}=3: 1$, and during the growth process, the hexagonal graphene domain is enclosed by the six h-BN epistrips with GBs formed between them (Fig. 4b2, b3), and the G-h-BN flake becomes a hexagon. Due to the unbalanced growth among these six $\mathrm{h}-\mathrm{BN}$ grains and the $\mathrm{R}_{\mathrm{ZZB}}$ being smaller than $R_{Z Z N}$, branches at the hexagon vertices are formed during further growth (Fig. 4b4), which is consistent with our experimental observations in Fig. 3b, c. If $R_{\mathrm{ZZN}}=$ $R_{Z Z B}$, during the growth process (Fig. 4c1-c4), a hexagonal G-h-BN flake is formed and enclosed by only ZZB edges. In addition, very small concave corners at the vertices of the flake are observed. A simple geometry analysis shows that formation of such hexagonal G-h-BN flakes with only ZZB edges requires $R_{Z Z N}: R_{Z Z B} \geq 1: 1$ (Figs. S15 and S16). Indeed, if we continuously increase $R_{Z Z B}$ with respect to $R_{Z Z N}$, ZZB edges in the G-h-BN flakes decrease in size, and ZZN edges appear at the concave corners (Fig. 4d1-d4). Eventually, the ZZB edges disappear, and the G-h-BN flake is only enclosed by ZZN edges (Fig. 4e1-e4). After straightforward analysis, we determine that steady state G-h-BN flakes, whose shape does not change after a long growth time and who have structures similar to that shown in Fig. 3i, appear if $R_{Z Z B}$ is two times of $R_{Z Z N}$ (see $S I$ for details). The G-h-BN flakes obtained from the above 


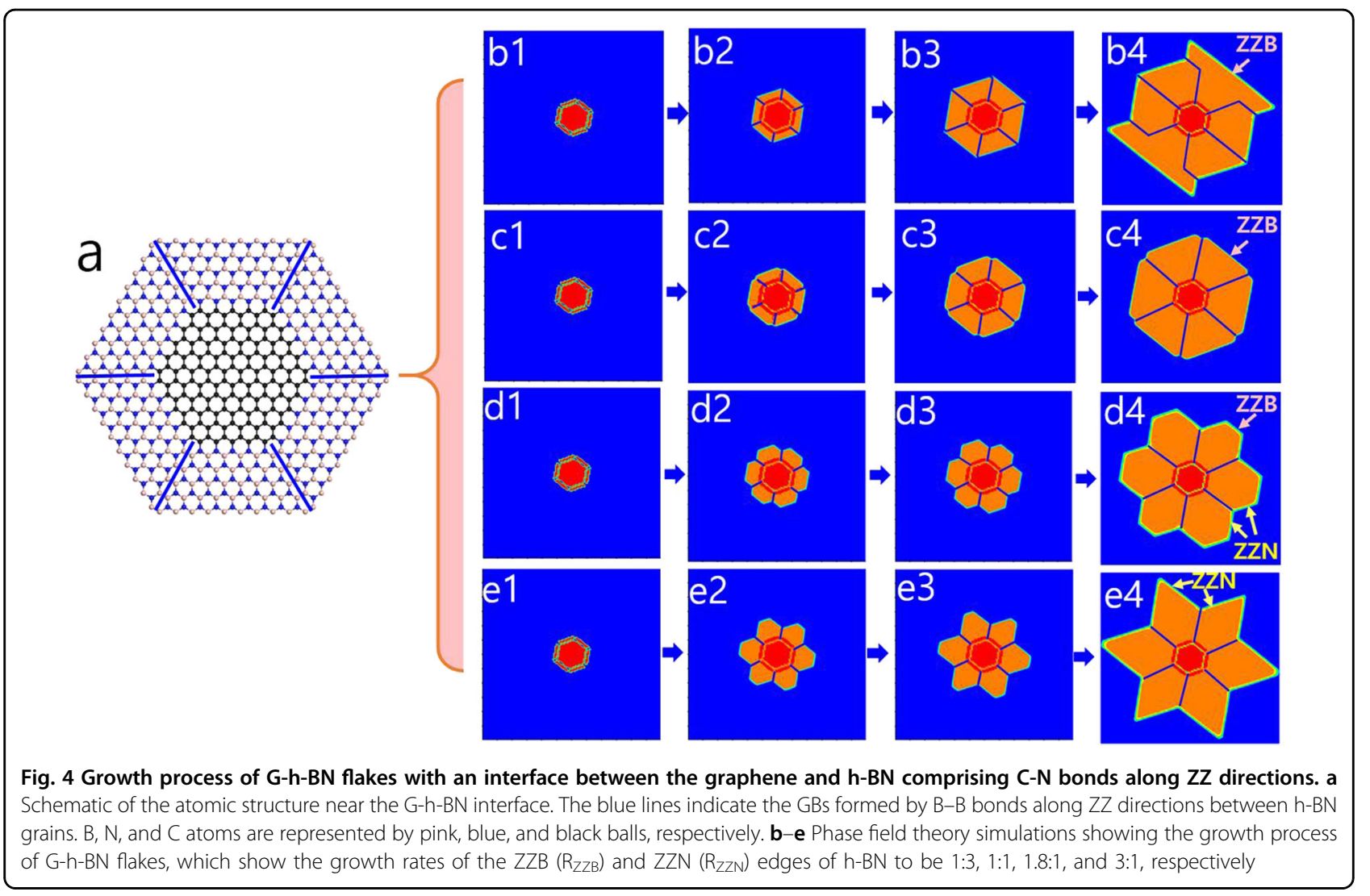

simulations under different conditions are very consistent with our experimental observations.

In the above PFT simulations, we assumed that the interface between the graphene and $\mathrm{h}-\mathrm{BN}$ is formed by $\mathrm{C}-\mathrm{N}$ bonds along the $\mathrm{ZZ}$ directions. However, the interface structure can vary with experimental conditions. Using density functional theory calculations, we compared the stability of a h-BN epistrip connected to the graphene $\mathrm{ZZ}$ edge with different configurations (Fig. 5a-h). Figure $5 \mathrm{i}$ shows the stability diagram of a h-BN epistrip connecting to a graphene $\mathrm{ZZ}$ edge. The outer edge of the $\mathrm{h}$-BN epistrip is passivated by hydrogen under high chemical hydrogen potential $\left(\mu_{\mathrm{H}}\right)$ and passivated by the $\mathrm{Cu}$ substrate under low $\mu_{\mathrm{H}}$. In addition, the interface structure between graphene and h-BN can also change with the chemical potential of nitrogen $\left(\mu_{\mathrm{N}}\right)$. Under low $\mu_{\mathrm{N}}$, the interface is formed by the graphene Klein edge and the h-BN Klein edge along the ZZN direction. With increasing $\mu_{\mathrm{N}}$, the interface is formed by the graphene $\mathrm{ZZ}$ edge and the h-BN ZZB edge. At high $\mu_{\mathrm{N}}$, the interface is formed by the graphene Klein edge and the h-BN Klein edge along the ZZB direction. It should be noted that forming a h-BN epistrip along the graphene $\mathrm{ZZ}$ edge is a kinetic process controlled by $1 \mathrm{D}$ nucleation and growth. Therefore, further experimental and theoretical work is needed on this point. It should be noted that the above discussion on the variation of G-h-BN flake shape based on PFT simulations only depends on the relative growth rates of h-BN ZZB and $\mathrm{ZZN}$ edges and thus is valid regardless of the interface type between graphene and h-BN epistrips.

\section{Conclusions}

In summary, highly confined growth of h-BN templated by graphene edges that forms lateral heterostructures is demonstrated. Precise control over the morphology of the as-grown G-h-BN flakes is realized by tuning the growth conditions; thus, three shapes of the flakes are prepared. The growth processes of the G-h-BN flakes with various shapes are simulated based on PFT and are consistent with the experimental observations. The controlled growth of an in-plane G-h-BN heterostructure with an engineered morphology and the understanding of the growth mechanism can accelerate construction of various heterostructures based on 2D materials for a wide range of applications.

\section{Methods}

Growth of G-h-BN lateral heterostructures

A two-step APCVD method was employed. Liquid $\mathrm{Cu}$ was used as the catalytic substrate, and methane $\left(\mathrm{CH}_{4}\right)$ and ammonia borane $\left(\mathrm{H}_{3} \mathrm{BNH}_{3}, \mathrm{AB}\right)$ were chosen as the precursors for graphene and h-BN growth, respectively. First, regular graphene hexagons were grown ca. $1100^{\circ} \mathrm{C}$ 

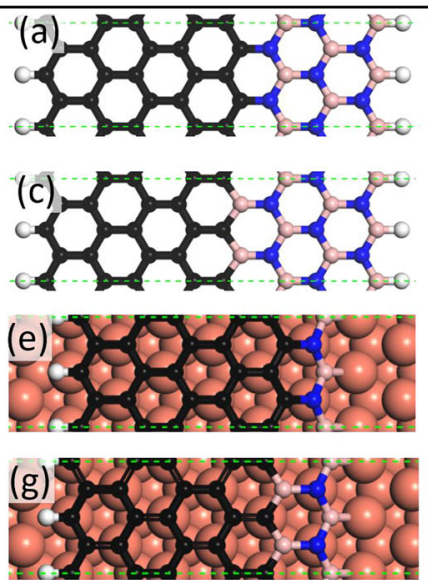
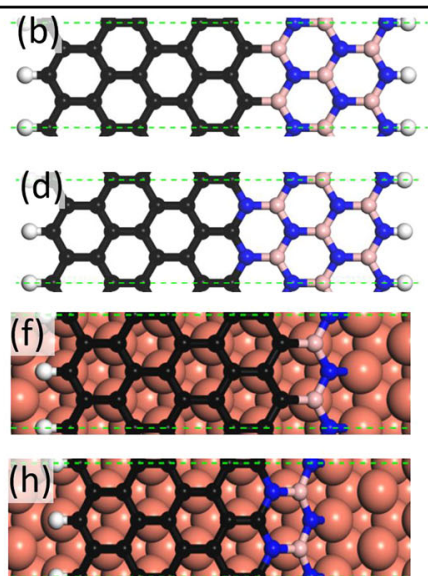

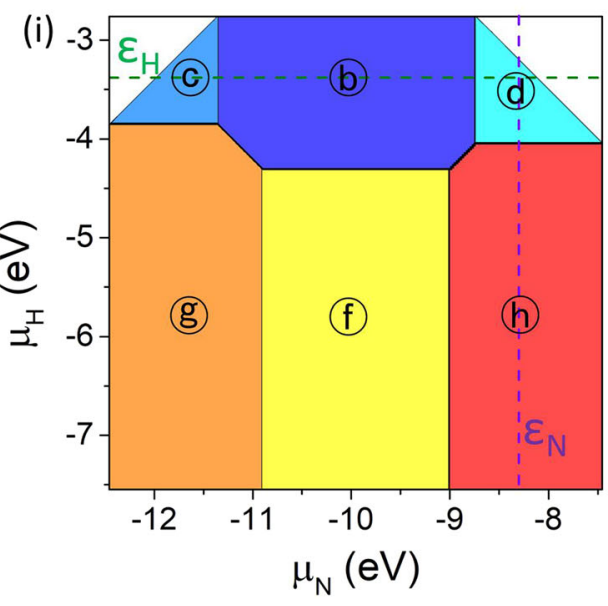

Fig. $\mathbf{5}$ a-d Structure of the h-BN epistrip connecting to a graphene ZZ edge with the outer edge passivated by hydrogen and $\mathbf{e}-\mathbf{h}$ the Cu substrate. Four different interface structures between graphene and h-BN are considered. i Stability diagram of a h-BN epistrip connecting to the graphene $\mathrm{ZZ}$ edge as a function of $\mu_{\mathrm{N}}$ and $\mu_{\mathrm{H}}$. The most stable h-BN epistrip structures are denoted by different colors. The energies of an $\mathrm{H}$ atom in an $\mathrm{H}_{2}$ molecule $\left(\varepsilon_{H}\right)$ and an $\mathrm{N}$ atom in $\mathrm{N}_{2}$ molecule $\left(\varepsilon_{\mathrm{N}}\right)$ are denoted by green and purple dashed lines, respectively. The white area in the diagram indicates that some of the formation energies of these eight h-BN epistrips are negative

on the liquid $\mathrm{Cu}$ surface under conditions of $3 \mathrm{sccm} \mathrm{CH}_{4}$ and $200 \mathrm{sccm} \mathrm{H}_{2}$ with a growth time of $5 \mathrm{~min}$. Next, the $\mathrm{CH}_{4}$ was stopped to terminate the growth of the graphene, and the $\mathrm{H}_{2}$ continued flowing. The $\mathrm{AB}$ source was then heated and rose to a steady-state value of $60-70{ }^{\circ} \mathrm{C}$ to induce sublimation. After the precursor precipitated on the liquid $\mathrm{Cu}$ surface, $\mathrm{h}-\mathrm{BN}$ nucleated, and there was a growth phase that lasted $5-8 \mathrm{~min}$, followed by rapid sample cooling.

\section{Transfer of G-h-BN samples}

A thin layer of poly(methyl methacrylate) (PMMA, weight-averaged molecular mass $\mathrm{Mw}=600,000,4 \mathrm{wt} \%$ in ethyl lactate) was first spin-coated on the surface at 5000 r.p.m. for $1 \mathrm{~min}$ and cured at $180^{\circ} \mathrm{C}$ for $30 \mathrm{~min}$; the PMMA-coated samples were then immersed in a $0.2 \mathrm{M}$ $\left(\mathrm{NH}_{4}\right)_{2} \mathrm{~S}_{2} \mathrm{O}_{8}$ solution at $70^{\circ} \mathrm{C}$ for $10 \mathrm{~min}$ to etch the $\mathrm{Cu}$ substrate. After that, the PMMA-coated G-h-BN flakes were stamped onto the $\mathrm{SiO}_{2} / \mathrm{Si}$ substrate, and warm acetone $\left(55^{\circ} \mathrm{C}\right)$ was used to dissolve the PMMA layer.

\section{Characterization of the G-h-BN samples}

Optical images were obtained using an Olympus BX51 microscope. SEM measurements were conducted on the FEI Verios 460 Field Emission Scanning Electron Microscope. AFM images were obtained using a Bruker Dimension FastScan atomic force microscope in tapping mode. Raman spectra were recorded at room temperature using a WITec Raman microscope with laser excitation at $532 \mathrm{~nm}$. For Raman mapping tests, the peak for h-BN, $E_{2 g}$ at $1371 \mathrm{~cm}^{-1}$ was obtained and analyzed.

\section{Acknowledgements}

This research is supported by A*STAR AME IRG A1783C0011 and IBS-R019-D1, and the computational resources from CMCM and IBS are also acknowledged.

\section{Authors contributions}

H.Y.Y. and D.C.G. conceived the idea, D.C.G. performed the growth experiments and materials characterization and wrote the paper. J.C.D. and F.D. performed the theoretical calculations. All authors discussed the results and commented on the manuscript.

Conflict of interest

The authors declare that they have no conflict of interest.

\section{Publisher's note}

Springer Nature remains neutral with regard to jurisdictional claims in published maps and institutional affiliations.

Supplementary information is available for this paper at https://doi.org/ 10.1038/s41427-019-0162-6.

Received: 19 March 2019 Revised: 26 June 2019 Accepted: 17 July 2019. Published online: 11 October 2019

\section{References}

1. Hitoshi, K., Tsunenori, S., Tetsuo, T., Eiichi, S. \& Kiyoko, N. Heteroepitaxial growth of GaP on a Si (100) substrate by molecular beam epitaxy. Jpn. J. Appl. Phys. 21, L68 (1982).

2. Pogge, H. B., Kemlage, B. M. \& Broadie, R. W. The heteroepitaxial growth of GaP films on Si substrates. J. Cryst. Growth 37, 13-22 (1977).

3. Han, G. H. et al. Continuous growth of hexagonal graphene and boron nitride in-plane heterostructures by atmospheric pressure chemical vapor deposition. ACS Nano 7, 10129-10138 (2013).

4. Kim, S. M. et al. Synthesis of patched or stacked graphene and hBN flakes: a route to hybrid structure discovery. Nano Lett. 13, 933-941 (2013).

5. Liu, Z. et al. In-plane heterostructures of graphene and hexagonal boron nitride with controlled domain sizes. Nat. Nanotechnol. 8, 119-124 (2013).

6. $\mathrm{Ci}$, L. et al. Atomic layers of hybridized boron nitride and graphene domains. Nat. Mater. 9, 430-435 (2010). 
7. Sutter, P., Cortes, R., Lahiri, J. \& Sutter, E. Interface formation in monolayer graphene-boron nitride heterostructures. Nano Lett. 12, 4869-4874 (2012).

8. Zhang, J. F., Xie, W. Y., Xu, X. H., Zhang, S. B. \& Zhao, J. J. Structural and electronic properties of interfaces in graphene and hexagonal boron nitride lateral heterostructures. Chem. Mater. 28, 5022-5028 (2016).

9. Liu, L. et al. Heteroepitaxial growth of two-dimensional hexagonal boron nitride templated by graphene edges. Science 343, 163-167 (2014).

10. Gao, T. et al. Temperature-triggered chemical switching growth of in-plane and vertically stacked graphene-boron nitride heterostructures. Nat. Commun. 6. e6835 (2015).

11. Lu, G. et al. Synthesis of high-quality graphene and hexagonal boron nitride monolayer in-plane heterostructure on Cu-Ni alloy. Adv. Sci. 4, 1700076 (2017).

12. Geng, D. C. et al. Self-aligned single-crystal graphene grains. Adv. Funct. Mater. 24, 1664-1670 (2014).

13. Geng, D. C. et al. Uniform hexagonal graphene flakes and films grown on liquid copper surface. Proc. Natl. Acad. Sci. USA 109, 7992-7996 (2012).

14. Dral, A. P. et al. Atomically defined templates for epitaxial growth of complex oxide thin films. J. Vis. Exp. 94, 52209 (2014).

15. Levendorf, M. P. et al. Graphene and boron nitride lateral heterostructures for atomically thin circuitry. Nature 488, 627-632 (2012).

16. Vlassiouk, l. et al. Role of hydrogen in chemical vapor deposition growth of large single-crystal graphene. ACS Nano 5, 6069-6076 (2011).

17. $\mathrm{Wu}, \mathrm{B}$. et al. Self-organized graphene crystal patterns. NPG Asia Mater. 5, e36 (2013).

18. Yu, Q. et al. Control and characterization of individual grains and grain boundaries in graphene grown by chemical vapor deposition. Nat. Mater. 10 443-449 (2011).

19. Geng, D. C., Wang, H. P., Yang, J. \& Yu, G. Controlled assembly of SiOx nanoparticles in graphene. Mater. Horiz. 3, 568-574 (2016).

20. Guo, W. et al. Governing rule for dynamic formation of grain boundaries in grown graphene. ACS Nano 9, 5792-5798 (2015).
21. Wu, B. et al. Equiangular hexagon-shape-controlled synthesis of graphene on copper surface. Adv. Mater. 23, 3522-3525 (2011).

22. $\mathrm{Ma}$, T. et al. Repeated growth-etching-regrowth for large-area defect-free single-crystal graphene by chemical vapor deposition. ACS Nano $\mathbf{8}$, 12806-12813 (2014).

23. $\mathrm{Ma}$, $\mathrm{T}$. et al. asEdge-controlled growth and kinetics of single-crystal graphene domains by chemical vapor deposition. Proc. Natl Acad. Sci. USA 110, 20386-20391 (2013).

24. Li, Q. C., Liu, M. X., Zhang, Y. F. \& Liu, Z. F. Hexagonal boron nitride-graphene heterostructures: synthesis and interfacial properties. Small 12, 32-50 (2016).

25. Zhao, J. J., Cheng, K., Han, N. N. \& Zhang, J. F. Growth control, interface behavior, band alignment, and potential device applications of 2D lateral heterostructures. WIREs Comput. Mol. Sci. 8, e1353 (2018).

26. Zhao, R. Q., Gao, J. F., Liu, Z. F. \& Ding, F. The reconstructed edges of the hexagonal BN. Nanoscale 7, 9723-9730 (2015).

27. Shu, H. B., Chen, X. S. \& Ding, F. The edge termination controlled kinetics in graphene chemical vapor deposition growth. Chem. Sci. 5, 4639-4645 (2014).

28. Kidambi, P. R. et al. In situ observations during chemical vapor deposition of hexagonal boron nitride on polycrystalline copper. Chem. Mater. 26 6380-6392 (2014).

29. Liu, Y. Y., Bhowmick, S. \& Yakobson, B. I. BN white graphene with "colorful" edges: the energies and morphology. Nano Lett. 11, 3113-3116 (2011).

30. Zhang, Z. H., Liu, Y. Y., Yang, Y. \& Yakobson, B. I. Growth mechanism and morphology of hexagonal boron nitride. Nano Lett. 16, 1398-1403 (2016).

31. Tay, R. Y. et al. Synthesis of aligned symmetrical multifaceted monolayer hexagonal boron nitride single crystals on resolidified copper. Nanoscale $\mathbf{8}$, 2434-2444 (2016)

32. Tay, R. Y. et al. Growth of large single-crystalline two-dimensional boron nitride hexagons on electropolished copper. Nano Lett. 14, 839-846 (2014). 